\title{
On the Timing of Discrete Events in Event-Driven Control Systems
}

\author{
Manel Velasco, Pau Martí, and Camilo Lozoya \\ Automatic Control Department, Technical University of Catalonia, \\ Pau Gargallo 5, 08028 Barcelona, Spain, \\ manel.velasco@upc.edu
}

\begin{abstract}
This paper presents an analysis method to determine offline at what intervals have to be taken the samples for various types of eventdriven control systems.
\end{abstract}

\section{Introduction}

For certain type of event-driven controllers and for time-driven controllers this paper shows that the distance covered by the system trajectory is proportional to the norm of the state. This property permits to determine the variations in the sampling times generated by discrete-events as a function of the state direction. For second order systems a geometric approach is proposed.

\section{Event-driven control systems model}

We consider the control system

$$
\begin{aligned}
& \dot{x}=A x+B u \\
& y=C x
\end{aligned}
$$

with $x \in \mathbb{R}^{n \times 1}, A \in \mathbb{R}^{n \times n}, B \in \mathbb{R}^{n \times m}, u \in \mathbb{R}^{1 \times m}$, and $C \in \mathbb{R}^{1 \times n}$. Let

$$
u_{k}=L x_{k}
$$

be the control updates given by a linear feedback controller designed in the continuous-time domain but using only samples of the state at discrete instants $t_{0}, t_{1}, \ldots, t_{k}, \ldots$. Between control updates, $u(t)=u_{k}$ in $t \in\left[t_{k}, t_{k+1}[\right.$.

\section{Analysis of various event conditions}

In event-driven control systems, event conditions are the controller execution rules. We analyze event conditions where samples are taken when some function of the system state exceeds a threshold, as in e.g. [1] or [2]. Let

$$
e(t)=x(t)-x_{k}
$$


be the error evolution between consecutive samples with $t \in\left[t_{k}, t_{k+1}[\right.$. In the approach presented in [1] the event condition is defined as

$$
\gamma_{k}:|e(t)|=\eta|x(t)|
$$

where $0<\eta \leq 1$. And in the scheme of [2], the event condition can be stated as

$$
\gamma_{k}: e_{k}^{T} M e_{k}=\eta x_{k}^{T} M x_{k}
$$

where $0<\eta \leq 1$ and $M \in \mathbb{R}^{n \times n}$.

In general, for some event-driven schemes, event conditions can be defined as

$$
\gamma_{k}: g\left(e(t), x_{k}, \eta\right)=0
$$

where $g(\cdot) \in \mathbb{R}$, and $\eta$ is a set of given parameters. We study whether time-driven control systems can be similarly specified. Let

$$
\begin{aligned}
x_{k+1} & =\Phi(t) x_{k}+\Gamma(t) u_{k} \\
y_{k} & =C x_{k}
\end{aligned}
$$

be the discrete-time system obtained by sampling (1) with period $t=h$, where

$$
\Phi(t)=e^{A t} \quad \text { and } \quad \Gamma(t)=\int_{0}^{t} e^{A s} d s B .
$$

From (3), (7), and (2) we observe that the event condition

$$
\gamma_{k}: e_{k}=(\Phi(h)+\Gamma(h) L-I) x_{k}
$$

triggers control updates at equidistant points in time, given by $h$.

For notation convenience, a vector $v_{k}$ will be denoted as

$$
v_{k}=r_{k}^{v}\left[\begin{array}{c}
\cos \theta_{k}^{v} \\
\sin \theta_{k}^{v}
\end{array}\right]
$$

where $r_{k}^{v}$ and $\theta_{k}^{v}$ are the modulus and angle of $v_{k}$.

Proposition 1. For two-dimensional systems described by (1)-(2), if control updates are triggered by event conditions (4), (5) or (8), it holds that

$$
\left\|e_{k}\right\|=\alpha\left\|x_{k}\right\| f\left(\theta_{k}^{x}, t\right)
$$

with $\alpha \in \mathbb{R}, f:\left[0,2 \pi\left[\rightarrow \mathbb{R}\right.\right.$, and $e_{k}$ given by (3).

Proof. Event condition (4) can be rewritten as

$$
e_{k}^{T} e_{k}=\eta^{2} x_{k}^{T} P(t) x_{k}
$$

where $P(t)=(\Phi(t)+\Gamma(t) L)^{T}(\Phi(t)+\Gamma(t) L)$. Eq. (11) in terms of (9) is

$$
\left(r_{k}^{e}\right)^{2}\left[\cos \theta_{k}^{e} \sin \theta_{k}^{e}\right]\left[\begin{array}{c}
\cos \theta_{k}^{e} \\
\sin \theta_{k}^{e}
\end{array}\right]=\eta^{2}\left(r_{k}^{x}\right)^{2}\left[\cos \theta_{k}^{x} \sin \theta_{k}^{x}\right] P(t)\left[\begin{array}{c}
\cos \theta_{k}^{x} \\
\sin \theta_{k}^{x}
\end{array}\right]
$$


which simplifies to

$$
r_{k}^{e}=\eta r_{k}^{x} \sqrt{\left[\cos \theta_{k}^{x} \sin \theta_{k}^{x}\right] P(t)\left[\begin{array}{c}
\cos \theta_{k}^{x} \\
\sin \theta_{k}^{x}
\end{array}\right]}
$$

From (8) with $t=h$, it follows that

$$
e_{k}^{T} e_{k}=x_{k}^{T} Q(t) x_{k}
$$

where $Q(t)=(\Phi(t)+\Gamma(t) L-I)^{T}(\Phi(t)+\Gamma(t) L-I)$. Then Eq. (14) simplifies to

$$
r_{k}^{e}=r_{k}^{x} \sqrt{\left[\cos \theta_{k}^{x} \sin \theta_{k}^{x}\right] Q(t)\left[\begin{array}{c}
\cos \theta_{k}^{x} \\
\sin \theta_{k}^{x}
\end{array}\right]}
$$

Similarly, condition (5) can be written as

$$
\left(r_{k}^{e}\right)^{2}\left[\cos \theta_{k}^{e} \sin \theta_{k}^{e}\right] M\left[\begin{array}{c}
\cos \theta_{k}^{e} \\
\sin \theta_{k}^{e}
\end{array}\right]=\eta\left(r_{k}^{x}\right)^{2}\left[\cos \theta_{k}^{x} \sin \theta_{k}^{x}\right] M\left[\begin{array}{c}
\cos \theta_{k}^{x} \\
\sin \theta_{k}^{x}
\end{array}\right]
$$

which reduces to

$$
r_{k}^{e}=\sqrt{\eta} r_{k}^{x} \frac{\left[\cos \theta_{k}^{x} \sin \theta_{k}^{x}\right] M\left[\begin{array}{l}
\cos \theta_{k}^{x} \\
\sin \theta_{k}^{x}
\end{array}\right]}{\left[\cos \theta_{k}^{e} \sin \theta_{k}^{e}\right] M\left[\begin{array}{c}
\cos \theta_{k}^{e} \\
\sin \theta_{k}^{e}
\end{array}\right]}=\sqrt{\eta} r_{k}^{x} g\left(\theta_{k}^{x}, t\right)
$$

The last equality, considering $R(t)=(\Phi(t)+\Gamma(t) L)$, holds because

$$
\theta_{k}^{e}=\arctan \left(\frac{y^{e}}{x^{e}}\right)=\arctan \left(\frac{r_{k}^{x}\left[\begin{array}{ll}
0 & 1
\end{array}\right] R(t)\left[\begin{array}{l}
\cos \theta_{k}^{x} \\
\sin \theta_{k}^{x}
\end{array}\right]}{r_{k}^{x}\left[\begin{array}{ll}
1 & 0
\end{array}\right] R(t)\left[\begin{array}{l}
\cos \theta_{k}^{x} \\
\sin \theta_{k}^{x}
\end{array}\right]}\right)
$$

Remark 1. Equations (13), (15), and (17) specify invariant boundaries for $\left|e_{k}\right|$ when for example a spheric parametrization of the unitary vector of the system state is used. These boundaries provide information about all possible covered distances by the system trajectory after the occurrence of an event.

\section{Geometric approach}

Since the derived boundaries scale on the norm of the system state, we can compare systems by geometrically mapping boundaries. Note that boundary (15) has constant period $h$. Therefore, solving (13) and (15) for $t$, or (17) and (15), we can determine the variations in sampling times generated by event conditions (4), (5), or any event-driven scheme whose event condition fulfills proposition 1 . The 

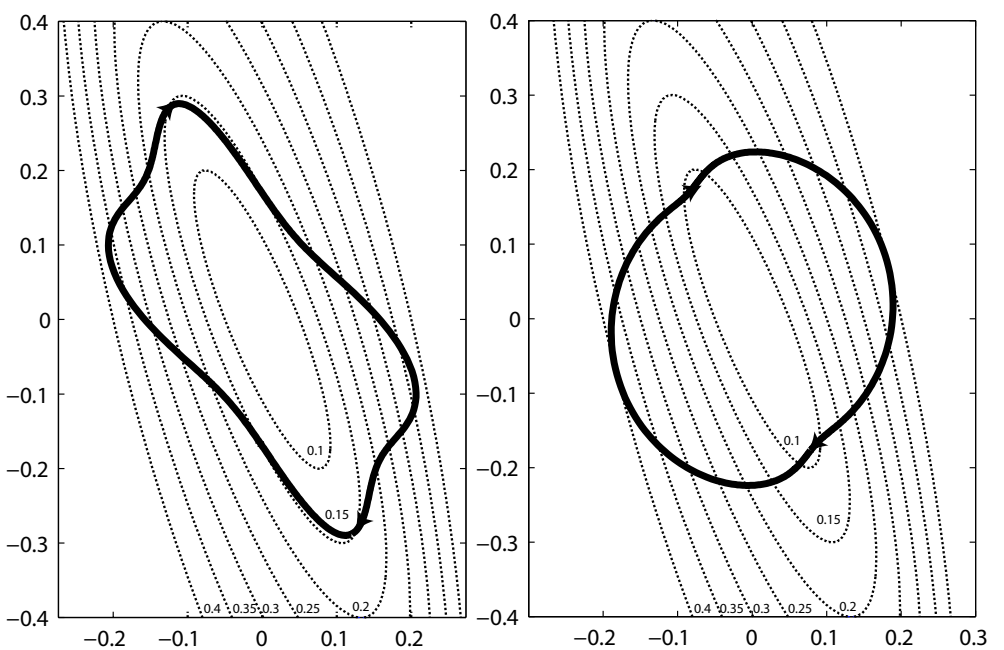

Fig. 1. Mapping of (17) (left) and (13) (right) on top of a time grid generated by (15)

mapping consists in plotting a time grid composed by boundaries generated by (15) with different periods. And on top of them, we plot the boundary generated by (13) or (17). Then by inspecting the superposition, we can directly assert the character of time between sampling instants that (4) or (5) generates.

As an example, consider that the double integrator system is controlled by (2) with $L=\left[\begin{array}{ll}1.0001 & 1.7322\end{array}\right]$, which can be obtained from eq. (1)-(6) of [2]. It is easy to verify that $L$ stabilizes the system when applied with event conditions (4), (5), or $(8)(\eta=0.5)$. In Figure 1 we plot the mapping for (17) (left) and (13) (right). The time grid has been generated using (15) for periods $h=0.1$ to 0.4. For example, by looking to the left sub-figure, we deduce that the maximum and minimum sampling interval are approximately $0.38 \mathrm{~s}$ and $0.15 \mathrm{~s}$, respectively.

\section{Conclusions and future work}

This paper has presented an analysis method that permits to study timing properties for various types of event-driven schemes. Future work will focus on finding the analytical solutions for the graphical method and it extension to an $n$-dimensional space. In additional, for schedulability of event-driven controllers and regulation of CPU load, it will be of interest to apply non-lineal techniques to study the nature of periods' dynamics.

\section{References}

1. Tabuada, P.: Event-triggered real-time scheduling of stabilizing control tasks. In IEEE Transactions on Automatic Control, 52(9), pp. 1680-1685 (2007)

2. Wang, X. and M. Lemmon: Self-triggered Feedback Control Systems with FiniteGain L2 Stability. Submitted to IEEE Transactions on Automatic Control (2007) 